\title{
Event Driven Service with Unified Identification for Next Generation Network
}

\author{
Dong-Il Kim, Soong-Hee Lee, Ki-Tae Kim, Member, KIMICS
}

\begin{abstract}
Event driven service (EDS) is one of such services administrating different functions of multiple service providers according to the user situation. This paper first describes the service model of EDS, a User-centric Service for multiple service provider environments over the next generation networks. The multiple provider environments stimulates the unified identifier management, namely unified identification (U-ID), to enable users to be provided network services without awareness of multiple providers. Combining these two concepts, i.e., EDS and U-ID, the designed structure of EDS with U-ID and related procedures are given. Finally, the implementation results tested on Korea Advanced Research Network (KOREN) are described.
\end{abstract}

Index Terms - Next Generation Network, EDS with U-ID, KOREN

\section{INTRODUCTION}

Next Generation networks will have functionalities to adapt to the user demands for various kinds of services as the future users may require network services customized to their needs. Moreover, they may want their own services, i.e., User-centric Services[1], for more flexibility just as shown in the current trends of Web 2.0, not a technology but a concept of usercentricity. However, current cases such as user-created contents (UCC) based on Web 2.0 concepts induce problems such as lack of business models, piracies over copyrights, etc. These problems are expected to be resolved by appropriate managements or regulations on the public basis by the future networks like NGN. Network and service providers may also be concerned about the situation that they might be excluded on the service exchanges between users.

Hence, it looks necessary to initiate to study on these issues to prepare a kind of framework enabling users to be provided their own services over future generation networks satisfying their demands while it helps providers to attain more business chances and practical regulations through controlled service components to avoid problems

\footnotetext{
Manuscript received July 1, 2010; accepted July 1, 2010.

Dong-il Kim is with the department of Information Communication Engineering, Dong-eui University(dikim@deu.ac.kr)
}

caused by user side. For this purpose, multiple network and service provider environment should be first considered for the successful deployment of the Usercentric Services over future networks.

Future network services should operate seamlessly across network infrastructures provided by multiple network and application service providers[2]. Seamless interconnection between networks managed by different network providers and seamless interoperability of services offered by different application service providers (ASPs) are required. Future networks will stimulate an evolution of the multi-service and multi-network provider environment or 'multi-provider' interoperable environment for open services.

There have been several efforts to deal with the issue of ID managements such as RFID, ENUM, and UCI, etc.[3 9]. Electronic or E.164 NUMber Mapping (ENUM) is defined by the Internet Engineering Task Force (IETF) in RFC 3761, that is the mapping of telephone numbers to uniform resource identifiers (URIs)[5]. The purpose of ENUM is limited as to enable the convergence between the PSTN and the Internet only. IETF RFC 4179 deals with universal content identifier (UCI) about the standardized structure of identification codes for relation between various digital contents[6]. These standards or studies only cover simple matches between limited types of identifiers for limited areas for a single provider that does not consider interactions between multiple service providers. Single sign on (SSO), defined in Y.IDMsec[7] for ID management in NGN, could manage multiple IDs in a unified way using unified login, but presents a limited feature for service provision while we must consider a unified ID management for User-centric Services in the wider range.

However, they are mainly related with web-based applications or mobile communications[10 12]. There had been no efforts to deploy the context aware service as one of the future User-centric Services before event driven service (EDS) was introduced in Y.Sup3[2] though NGN is regarded as the main infrastructure for future ubiquitous environment.

Event driven service will emerge as an important business model in the future network environments, e.g., $\mathrm{UBcN}$ (ultra broadband convergence network) in Korea. Hence, much effort needs to be concentrated into establishing the new concepts of User-centric Service via research and standardization activities. Moreover, we need to explore technical basis on unified management 
of identification, certification, and profile management to provide the User-centric Services. Therefore, the testing and verification process is required for confirmation after applying the developed results into the actual network. This leads to the verification tests using the network, that is similar with the future networks, and applying the User-centric Service scenario, that is expected to be applied into the future generation networks. Adapting and applying Korea Advanced Research Network (KOREN) for this purpose of verification tests is very important in that KOREN is the very infrastructure for testing $\mathrm{UBcN}$, that will be deployed in the future, and the most appropriate testing environment consequently.

The applied scheme and the results after implementation are explained and the conclusion is lastly given.

\section{EVENT DRIVEN SERVICE WITH UNIFIED IDENTIFICATION}

\subsection{Service model of EDS in ITU-T}

Event driven service (EDS) is regarded as a typical User-centric Service administrating functions owned by multiple service providers according to the user situation[2]. EDS provides users with the user-centric information and the communication by the various personal conditional environments. An alternative terminology for events for network-related applications is 'triggers' - these are assumed to be included in the present description.

Events could be defined as any activities which occur within a service area caused by user actions, connectivity signals, signals related to service selection and service availability, signals classifying provider and associated control signals, and billing signals. Events can be managed, for example, as part of a context-aware or user-centric application. EDS could carry out a procedure for processing user's requested information about mobile network-based entities, such as the combination with various applications, such as a location based service (LBS) and area information. In the future network environments, the user can be serviced his/her customized EDS in the network. The EDS automatically connects the appropriate terminal equipments (TEs) consistent with a user profile. EDS can also send the collected information to other requesting users by way of event information and using the procedures of combination with other services.

The user can record/retrieve the user-centric information to/from the information base through EDS. The information base may initially reside in the server of the provider side. The cache of the CTE may partly store the information and can be autonomously updated.

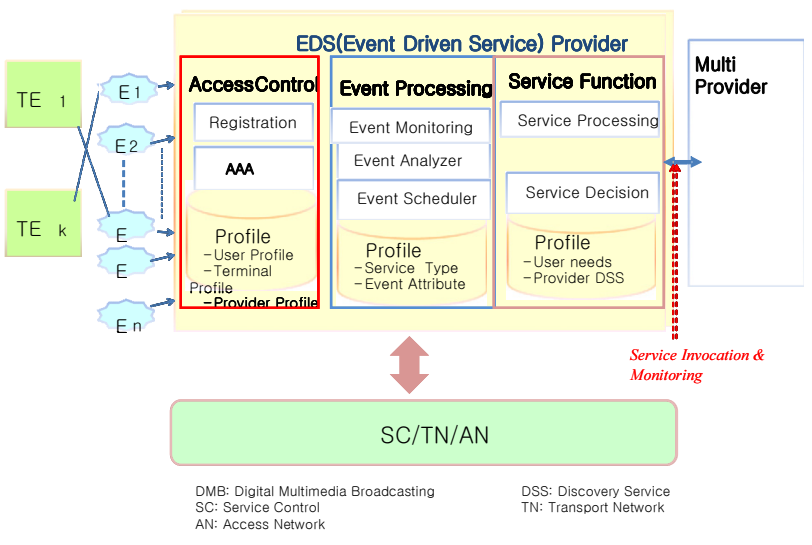

Fig. 1. Service model of the event driven service.

EDS consists of Access Control Function, Event Processing Function, and Service Function as shown in Fig. 1[2]. The services to be provided include LBS (Location Based Service), DSS (Discovery Service), and Push service, etc. in the multi-provider environment. $\mathrm{E}_{1}$, $E_{2, \ldots}, E_{n}$ refer to events or triggers.

Access Control function covers registrations of user profiles, terminal profiles, and provider profiles for providing the proper application services with applying AAA to users. The user requesting services could be offered with performing AAA function on the basis of preregistered profile data such as user ID, and Terminal ID, etc. These multiple IDs used in the profiles demand a more efficient method for manageable service provision that naturally leads to the introduction of a Unified ID management system in the next chapter.

Event Processing function makes the choice of the best suited access option, by taking into consideration of the application requirements and user preferences by analyzing event attributes. All this information is maintained and structured in profiles, which are located in the Access Profile of the Access Control. These profiles can be accessed by the service function in order to combine link-specific parameters with user preferences and application requirements for user-centricity.

\subsection{Service scenario of EDS with U-ID}

EDS can provide the user-centric services to users by efficiently managing the resources or functions residing in the multiple providers (ASPs or carriers). One of the methods required to achieve this goal is the unified ID management system, called U-ID in this paper. Event Driven Service (EDS), combined with Unified ID management (U-ID), i.e., EDS with U-ID is described.

U-ID(Unified Identification). U-ID can function as a service administrator for offering User-centric Services. Users can request services to service providers via the identifier that was granted for use by all service providers 
through unified authentication, accounting, and profile management. Service providers provide the requested service to the user after coordinating between themselves based on the identifier and related information. U-ID carries out ID management including unified authentication, unified accounting, and unified profile management between service providers. U-ID needs to communicate with EDS functions and unification profiles for the required service functions. U-ID can be applied to the profile-related procedures for the service provision such as EDS.

EDS with U-ID. EDS requires technologies that senses and analyzes events that happen on the real time basis, which provides services as the responses to the analyzed events. With the aid of U-ID, EDS enables User-centric Services between various networks and providers, which provide the customized services to the users and the enterprise environments on the real time basis, etc. Fig. 2 shows the interactions required for EDS with U-ID. On detecting event around users or terminals, the EDS server checks whether the events are registered or not by asking to the U-ID server. The EDS server coordinates between the U-ID server and users, between the U-ID server and service providers, or the U-ID server and service providers.

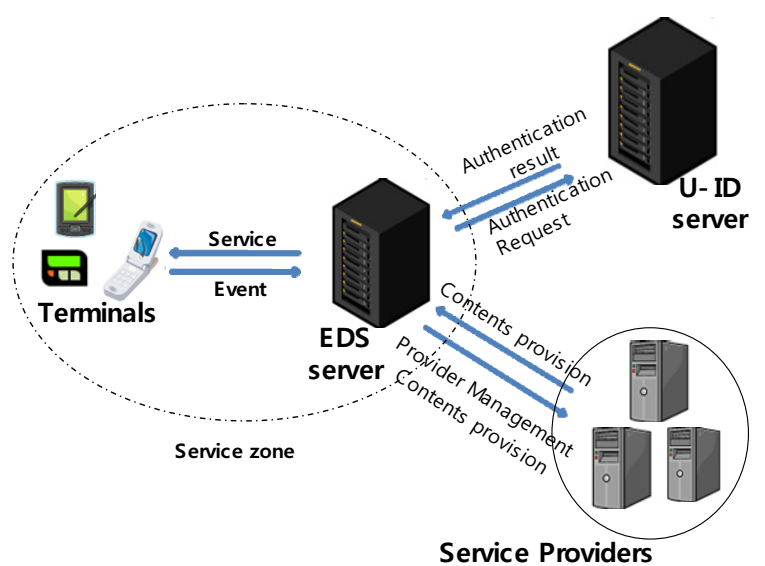

Fig. 2 Interactions for EDS with U-ID

EDS is provided combining the functions listed in Fig. 1, i.e., access control, event processing, and service function.

Access Control. This function requests the authentication for the access within the service area and manages the attached terminals. If attached terminals enter the service area without the users' requests, they will be automatically managed using the access codes in the terminals. For registration, terminals or users that entered service area are registered into the Access Control system. This process is carried out without access requests for immediate reaction to the possible occurrence of events. For authentication and/or authorization, the appropriate rights are authorized according to the information that passed U-ID authentication process. The personal information that passed U-ID authentication is analyzed and used to authorize the range of service rights that fits to the service user level and system capacity. For managing profiles, User Profile that passed U-ID authentication is registered to enable the efficient user management and service. Terminal Profile registered to the current service area is registered to enable the analysis of available services. For example, a terminal that supports not DMB but WLAN will broadcast the intended contents via IP streaming service.

Event Processing. All events occurring within the service area are managed and controlled applying this function. This function performs event-related procedures as the followings. For Event Monitoring, user/terminalrequested or registered events are monitored and delivered to the manager on the real-time basis. For Event Analysis, events are analyzed to investigate the current status or trigger service providing. Events caused by users are statistically analyzed and reported to the manager that will appropriately cope with the upcoming events using the analyzed result. For Event Scheduling, events are scheduled to be provided to users for more efficient processing. Events caused by users are scheduled to enable users to efficiently manage their own events. For Profiles, there are two types of event attributes to be stored in the profile, one for service and another for billing as follows: TYPE I (Service) profile stores the service features provided to the users; TYPE II (Billing) profile stores the billing information for services provided to the users.

Service Function. To provide more efficient services to users, user and terminal profiles are analyzed. Service providers are also managed. Service function includes the following procedures. For Service Processing, users and providers are linked and managed to prevent duplicate or incorrect service providing with this function. Providers for the same service or user-requested service are managed to guarantee service reliability to the users. In addition, services that were already in provision are analyzed and services are provided to enable users to actively interact (e.g., Push service). For Service Decision, services requested by users are linked with providers which can provide the required service. The priority or popularity can be used to choose the most appropriate service providers. For Profiles, there are profiles for user need and provider DSS as follows: User Need Profile stores services selected by users or provided to users that are used for services such as Push service; Provider DSS Profile manages a kind of directory in which service provider registered.

Service Control, Invocation and Monitoring. The services are controlled during the service provision. The efficient and easy management of QoS is possible by managing the provided services, terminals, and networks. It is especially needed for the case that multiple users demand the same service or that a user is provided 
services simultaneously. Services selected and requested by users are invocated and monitored.

\section{DESIGNED SERVICE STRUCTURE AND IMPLEMENTATION}

The EDS with U-ID is designed on the structure and procedure level applying the service scenario described in chapter 2. The details of the designed structure and procedures are explained in this chapter.

\subsection{Designed Service Structure of EDS with U-ID}

The service structure for EDS with U-ID is designed to consist of two parts, U-ID system and EDS system. We designed each system and considered interaction between two systems. The designed U-ID system roughly consists of two parts, a U-ID manager and the unification profile. Fig. 3 and 4 show the structure of the U-ID manager, and the structure of the unification profile each.

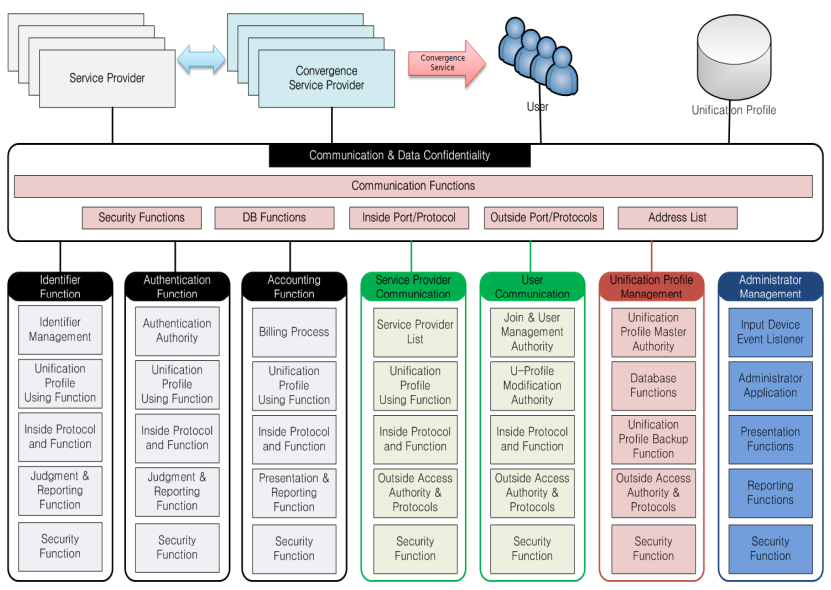

Fig. 3 Structure of the designed U-ID manager.

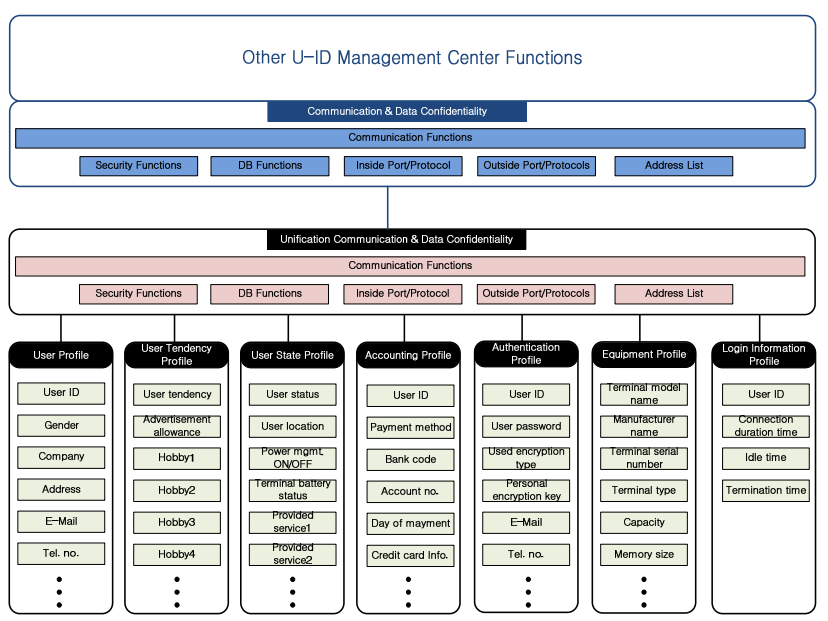

Fig. 4 Structure of the unification profile.
As shown in Fig. 3, the U-ID manager is composed of eight parts, Identifier Functions, Authentication Functions, Accounting Process, Administrator Management, Unification Profile Management, User Communication Management, Service Provider Communication Functions, and Communication \& Data Confidentiality. The Unification Profile includes User Profile, User Tendency Profile, User State Profile, Accounting Profile, Authentication Profile, Equipment Profile, and Login Information Profile, as shown in Fig. 4.

\subsection{Implementation of EDS with $U-I D$}

The designed structure needs to be verified through implementation and actual deployment. One of the practical ways to achieve this goal is to implement the service systems according to the designed structure as brief as possible and applies to the network that is possibly closest to the future network environments. We implement the EDS system applying the architecture of the implemented software shown in Fig. 5. The implemented software consists of the EDS system and the U-ID system communicating each other for exchange of identification and billing data.

Based on the architecture in Fig. 5, the structure of the profile and communication protocol is designed and the software for EDS with U-ID is implemented using SQL Database, Visual $\mathrm{C}++$, etc. as follows:

Event detection and service information output. It is required to detect events from the user side, demands authentication, and output service information about the authenticated user.

Storing, lookup, and modification of EDS server information. It is required to store, lookup, and modify information from/to EDS server.

Access to U-ID server. It is required to access U-ID server if needed, especially when the user authentication is required or requested. $\mathrm{D} / \mathrm{B}$ information for U-ID is updated in this function.

Storing, lookup, and modification of U-ID server information. It is required to store, lookup, and modify information from/to U-ID server for the purpose of registration of new users or terminals, authentication and authorization for the registered users or terminals, monitoring, etc.

Access to EDS server. It is required to access EDS server, when the user authentication is required or requested. Registration for new users and D/B for EDS update are also carried out. 


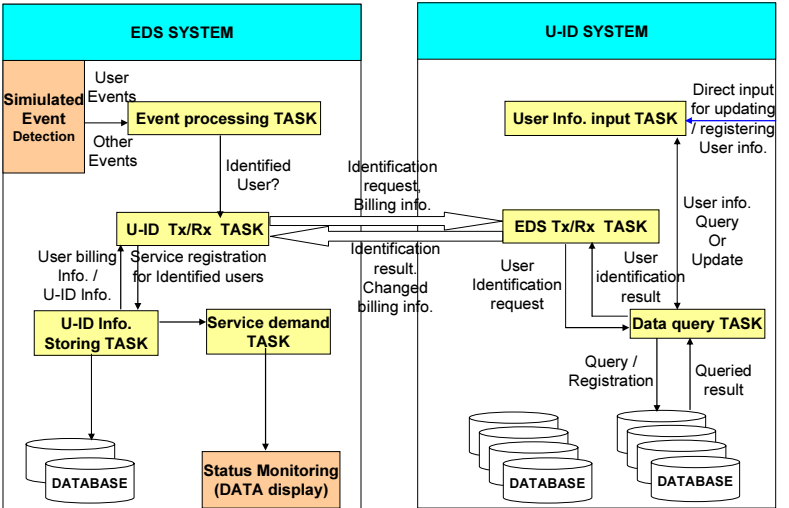

Fig. 5 Architecture of the implemented software for EDS with U-ID

\subsection{Testing and Verification of EDS with U-ID}

The testing and verification process is required for confirmation after applying the developed results into the actual network. This leads to the verification tests using the network, that is similar with the future networks, and applying the User-centric service scenario, that is expected to be applied into the future networks. Adapting and applying Korea Advanced Research Network (KOREN) for this purpose of verification tests is very important in that KOREN is the very infrastructure for testing $\mathrm{UBcN}$, that will be deployed in the future, and the most appropriate testing environment consequently. The implemented result was verified through interworking tests that was carried out in Inje University site and Dongeui University site, between which KOREN is linked. Inje University site manages a U-ID management server, unified profile, and a client. And Dongeui University site manages an EDS management server, EDS database, and a client. Fig. 6 shows the configuration of the testbed for interworking test though KOREN.

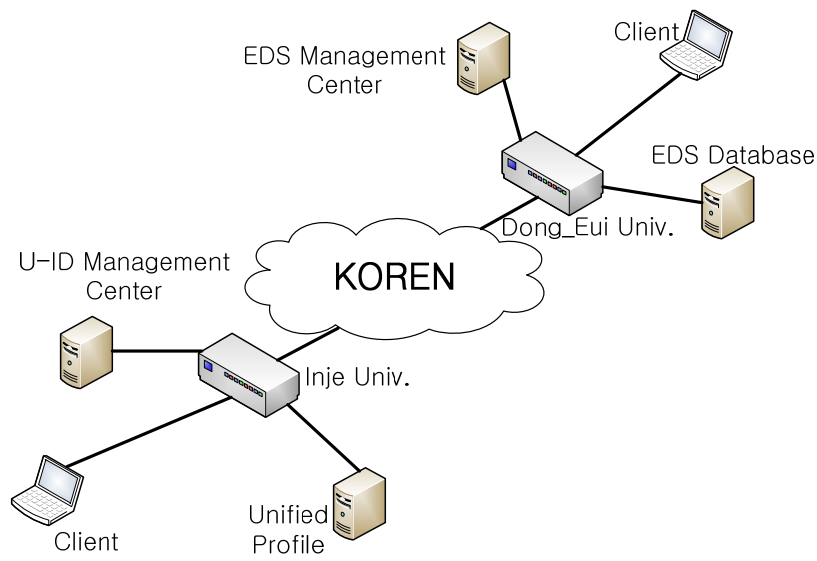

Fig. 6 Configuration of the testbed for interworking tests on KOREN.

\section{CONCLUSION AND FUTURE WORKS}

Researches on a User-centric Service over future networks, EDS with U-ID, and implementation and testing via KOREN were carried out by the participating members of the universities and organizations. More specifically, architecture and roles of EDS with U-ID, unification profiles were studied, designed and implemented to be tested in KOREN environment. The studied results were presented as the contributions in the international standardization meetings such as ITU-T.

The proposed service scenario includes the functional architectures and main functions for EDS with U-ID. Management software and unified profiles were established for interworking tests of EDS with U-ID in the KOREN environment. KOREN was used as a substitute for future networks in this test. The designed service structure and functions need to be studied for more scalability.

\section{ACKNOWLEDGMENT}

This work was supported by Dong-Eui University Grant ( 2010AA172)

\section{REFERENCES}

[1] Yong Zhang, et. al.: User-Centric Service Provision Model for Adaptive Ubiquitous Computing Applications, International Conference on Systems, Man and Cybernetics (2006)

[2] ITU-T Y.Sup.3: ITU-T Y.2000 series - Supplement on service scenarios for convergence services in a multiple network and application service provider environment", ITU-T Y.2000 series Y.Sup.3, ITU-T (2007)

[3] Young-gil Kim: Data transmission in u-ID sensor network configuration using Bluetooth, ICKIMICS 2004 Proceedings, KIMICS (2004)

[4] Sang-chul Shin, et. al.: Strategy for international standardization and wide spread of RFID/USN, KICS Journal, KICS (2004)

[5] IETF RFC 3761: The E.164 to Uniform Resource Identifiers (URI) Dynamic Delegation Discovery System (DDDS) Application (ENUM), IETF RFC 3761, IETF (2004)

[6] IETF RFC 4179: Using Universal Content Identifier (UCI) as Uniform Resource Names (URN), IETF RFC 4179, IETF (2005)

[7] ITU-T Y.IdMsec: NGN IDENTITY MANAGEMENT SECURITY, ITU-T Y.IdMsec, ITU-T (2007)

[8] Spyridon Vassilaras et. al.: Architecture Framework for Device Single Sign On in Personal Area Networks", Lecture Notes in Computer Science, Springer (2006)

[9] Tae-shik Sohn, et. al.: Single Sign-On System enabling Mutual Authentication in Multi Domain Environments, KISS Journal (2001)

[10] B. Schilit, N. Adams, and R. Want: Context-aware computing applications, IEEE Workshop on Mobile Computing Systems and Applications (WMCSA'94), Santa Cruz, CA, US, pp. 89-101 (1994)

[11] Rosemann, M., and Recker, J.: Context-aware process design: Exploring the extrinsic drivers for process flexibility, 18th international conference on advanced information systems engineering proceedings of workshops and doctoral consortium, pp. 149--158. Namur University Press, Luxembourg (2006)

[12] Soraya Kouadri Most'efaoui, B'eat Hirsbrunner: Context Aware Service Provision, Proceedings of the IEEE/ACS International Conference on Pervasive Services (ICPS'04), IEEE Computer Society (2004) 


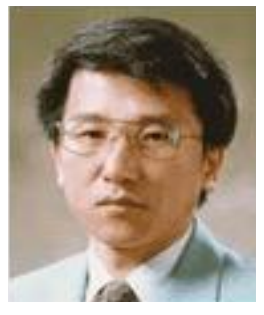

Dong-il Kim Received the Ph.D degree in electronics information and communication engineering from Kwang-woon University, Korea, in 1992. Since 1991, he has been at Dong-Eui University, Korea, where he is a professor at the information and communication engineering department. From 1983 to 1991, he was a general manager at switching research center in LG information and communication institute. He was a visiting researcher at standardization research center, ETRI(Electronics and Telecommunications Research Institute, Korea), from 1998 to 1999 and also a chief of computer center at DEU, from 2003 to 2007 . He is currently a director of KIMICS, member of international journal committee in KICS(Korea Institute of Communication Sciences), and international expert, the field of IT, in MIC(Ministry of information and Communication, Korea). His research interests are analysis of performance in a communication networks, protocols in wireless networks and standardization of ICT

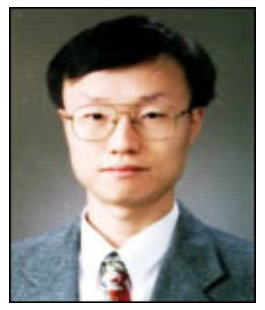

Soong- Hee Lee Received the B.S., M.S., and Ph.D. degrees from Kyungpook National University, Daegu in 1987, 1990, and 1995, respectively. From 1987 to 1997, he was a member of research staff in Electronics and Telecommunications Research Institute. Since 1997, he has been with the Dep't of Information and Communications Engineering, Inje University as an associate professor. His research activities are in the area of next generation network technologies and services. He was designated as an IT Standard Expert on behalf of Korea by the Ministry of Information and Communications in 2001. He is an Editor in the ITU-T SG13 and a Member of KIMICS.

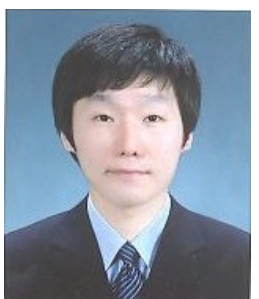

Ki-Tae Kim Received the B.S degree in information and communication engineering from Dong-eui University, Korea, in 2010. He's currently pursuing M.S degree in information and communication engineering at Dong-Eui University, Korea. His research interests are analysis of performance in a communication networks, protocols in wireless networks and Ubiquitous Sensor Network of ICT 